\section{Die psychiatrische Begutachtung}

\section{Eine allgemeine Einführung}

Ulrike Hoffmann-Richter

Stuttgart: Thieme; 2005.

Im Thieme-Verlag ist ein lesenswertes neues Buch über die psychiatrische Begutachtung im Auftrag von Versicherungen, speziell von Sozialversicherungen, erschienen.

Die Autorin, Ulrike Hoffmann-Richter, Leiterin des versicherungspsychiatrischen Dienstes der SUVA, beschreibt die wesentlichen Voraussetzungen für eine gelungene Begutachtung im Rahmen von Versicherungen. Sie konzentriert sich dabei auf das, was man gemeinhin als Prozessqualität bezeichnet, speziell auf Indikation und Ziel der Begutachtung, sowohl, was die verschiedenen Versicherungsarten betrifft, Kranken- und Unfallversicherung, Haftpflicht-, Arbeitslosenversicherung, Sozialhilfe, Opferentschädigung und weiteres, als auch, was die Unterschiede in den verschiedenen Rechtssystemen der deutschsprachigen Länder anbelangt. Eingehend widmet sie sich der Gutachter-Klienten-Beziehung, dem Interview und der Exploration, und dann vor allem der Datengrundlage und Methodik allgemeiner medizinischer und speziell psychiatrischer Untersuchungen, einschliesslich zusätzlicher Abklärungen wie bildgebende Verfahren, Fragebögen und Tests, neuropsychologische Testuntersuchungen. Besonders interessant sind die Ausführungen zur Dokumentation im Gutachten und zu den Lesarten dieser Dokumentation, ihre Textgattung und ihren Grad der Formalisierung. Der Hinweis auf die Bedeutung der Leserin eines Gutachtens und die Überlegungen zu ihrer spezifischen Aufmerksamkeit bei der Lektüre, aber auch über das Vorhandensein allfälliger Hintergrundannahmen aus dem Alltagswissen sind höchst interessante Aspekte, auf die in einem adäquaten, lesbaren Umfang eingegangen wird.

Das Kapitel über die Diagnose widmet sich eingehend dem Krankheitsbegriff, den Krankheitskonzepten, ihren Grenzen, aber auch ihren Möglichkeiten. Die Diagnosestellung wird als abhängig von der Erfahrung der Diagnostiker, von der Situation, der aufgewendeten Zeit und dem Ziel dargestellt. Für den Gutachter besonders wichtig sind sodann die Ausführungen zur Übersetzungsarbeit medizinischer Sachverhalte in juristische Begriffe. Hier versteht es die Autorin, die entscheidenden Fragestellungen in den verschiedenen Rechtssystemen der deutschsprachigen Länder zu beleuchten und gleichzeitig auch auf Interpretationsfallen, sei es auf Fallen in der Denk- und Übersetzungsarbeit oder auf
Fallen aus Hintergrundannahmen, hinzuweisen. Die praktischen Hinweise zur Erstellung des Gutachtens lassen sich als Art Checkliste verwenden, damit nichts vergessen geht. Angeschlossen wird ein Kapitel über Textstruktur, Autorschaften, aber auch über das Spannungsverhältnis zwischen Subjektivität und Objektivität und daraus sich ergebender Schlussfolgerungen für das Gutachten. Interessant und hilfreich ist der Umstand, dass auch an den Leser eines Gutachtens gedacht wird, an den Akt des Lesens, welcher sich als komplementäre Aufgabe zum Akt des Schreibens versteht.

Last but not least lockern verschiedene Illustrationen von Anna das Lesen des Buches in anregender Weise auf.

Wer glaubt, es handle sich um ein weiteres vergleichbares Werk in der deutschsprachigen Fachliteratur, welches sich mit Fragen der Begutachtung zuhanden von Versicherungen auseinandersetzt, täuscht sich. Ebenso täuscht sich, wer dem Untertitel einer allgemeinen Einführung folgend glaubt, sich auf ein Werk mit allgemein bekannten Grundsätzen der psychiatrischen Begutachtung einzulassen. Titel und Untertitel des Buches machen auf Understatement und lassen nicht von vornherein erkennen, welche grundlegenden Fakten und Fragen im Zusammenhang nicht nur mit psychiatrischer, sondern medizinischer Begutachtung überhaupt erörtert werden. Die Autorin stützt sich dabei auf eine ausserordentlich breite Palette medizinischer und nicht medizinischer Literatur. In ihrer Einführung bemerkt sie, dass es in der Medizin Fragen gibt, die nicht ausschliesslich naturwissenschaftlich angehbar sind und die auf das Fachgebiet der Psychiatrie projiziert werden: die Frage nach den theoretischen Konzepten, nach dem Bezug zwischen Grundlagenwissenschaften - wie Biologie, Physiologie, Biochemie, aber auch Soziologie, Psychologie, Kulturanthropologie - und klinischer Medizin und zwischen epidemiologischen, statistischen, empirischen Ergebnissen und individuellem Explorand oder Patientin. Sie entdeckt in geisteswissenschaftlichen Arbeiten höchst faszinierende Reflexionen über Medizin und Naturwissenschaft. Es gelingt der Autorin, als kommentierende und reflektierende Beobachterin und Teilnehmerin die reichhaltige Literatur zu einem aufschlussreichen, informativen Ganzen zu führen. Besonders bemerkenswert ist zudem die Tatsache, dass die Autorin die Fülle theoretischer Voraussetzungen mit den Anforderungen im praktischen Begutachtungsalltag zu verbinden weiss und damit dem Leser sowohl theoretische Grundlagen wie praktische Anleitung liefert und ihn gleichzeitig zwingt, über das eigene Tun, theoretisch, methodisch und praktisch, nachzudenken. Am Schluss bleibt das Gefühl, viel Wissenswertes erfahren zu haben, und zwar in Form einer gelungenen, umfassenden und trotzdem überblickbaren und lesbaren Synopsis. Für jeden und jede, der oder die sich mit medizinischen Gutachten beschäftigt, ob Medizinerin oder Jurist, zweifellos ein Muss.

Dr. med. Renato Marelli, Basel

\section{Die biopsychosoziale Dimension in der Medizin}

\section{Einführung in die biopsychosoziale Medizin}

\section{Rolf $\mathrm{H}$. Adler}

Stuttgart: Schattauer; 2005.

«Die Biomedizin hat segensreiche Fortschritte erzielt, aber auch in Sackgassen geführt, nämlich zu einer Medizin für Körper ohne Seelen und einer für Seelen ohne Körper», zitiert Rolf Adler Thure von Uexküll (1908-2004) am Anfang seines Buches. Und wenn er weiter schreibt: «Das biomedizinische Konzept deutet Krankheiten als Störungen einer komplexen (menschlichen) Maschine, ohne Berücksichtigung psychischer und sozialer Einflüsse», weiss man schlagartig, warum dieses Buch nicht nur wichtig sein könnte: Warum es notwendig ist.

Erklärungsmodelle von Krankheiten, die psychische und soziale Einflüsse draussenlassen - das tönt nach Anachronismus. Aber es ist heute, 2005, kein Anachronismus - es ist weitpraktizierte Spital- und Praxiswirklichkeit. Und Gutachterwirklichkeit: Keiner weiss es besser als Prof. em. Rolf Adler, der das biopsychosoziale Denken und Handeln einer ganzen Ärztegeneration als charismatischer Chefarzt des C.-L.-Lory-Hauses (1978-2001) entscheidend geprägt hat und in der Weiterbildung von Ärztinnen und Ärzten noch immer eine wegweisende Instanz ist.

Er hat in seiner schmalen «Einführung in die biopsychosoziale Medizin» (110 Seiten) ein Konzentrat von Grundbegriffen und Anleitungen zum praktischen Vorgehen geschaffen, das so gesammelt, übersichtlich und einleuchtend daherkommt (das Schwierigste ist, das Schwierige auf seine Einfachheit zu reduzieren!), dass das Lesen eine Freude ist. In einer Einführung soll man sich nicht verlieren - in einer Einführung soll man rasch auf das Wesentliche stossen und das Wesentliche entschlüsseln können - genau das geschieht in diesem Buch: Aufbau und Gliederung sind straff, klar geordnet, überschaubar.

In konzentrierten, knappen und bestechend klaren Sätzen erfährt man, was man zu wissen glaubte und doch nur ungenau gewusst hat, zum Beispiel Gewichtung und Ausdruck der 
Entwicklungslinie der ersten Lebensmonate und -jahre für die Prägung der individuellen Wirklichkeit des Patienten, der eine Kernbedeutung zukommt und die sich nur dem einfühlsamen Arzt öffnet. Oder die genaue Erklärung körperphysiologischer Prozesse als Ausdruck von Gefühlszuständen und Belastungsfaktoren: der vereinfachenden Deutungspsychologie steht hier die unerbittlich genaue, deduktive Prozessanalyse somatopsychisch-psychosomatischer Störungen des analytisch und somatisch Erfahrenen gegenüber.

Und natürlich steht - es kann, wenn das Buch von Rolf Adler stammt, nicht anders sein - der Mensch, der einzelne Mensch stets im Mittelpunkt von Theorie und Praxis: der verletzte und der verletzbare Mensch - und der unverletzte, aber verletzbare Arzt. Die Faszination des Interesses, die Sorgfalt und das Verstehen des kranken Menschen - und die Gefühle des Arztes, der dem Patienten gegenübersitzt und deren genaue Wahrnehmung notwendig ist, um das Geheimnis des Patienten zu ergründen (Gegenübertragung).

Es hat viele schöne, glasklar geschnittene Sätze in diesem Buch, die man mehr als einmal lesen muss. Zum Beispiel der Satz über die Empathie: «Empathie therapeutisch richtig eingesetzt heisst, sich nur phasenweise in den Patienten zu versetzen, qualitativ mitzuerleben, aber nicht quantitativ, sonst wird der Arzt in die Strudel der Gefühle des Patienten gerissen und verliert die Übersicht. Nur das Pendeln zwischen Einfühlen und distanzierter Position ist von Nutzen...»

Das ist ein wunderschöner Satz, der in wenigen Worten das umreisst, worüber schon ganze Symposien durchgeführt worden sind (er hätte auch Willi Henneler gefreut, den langiährigen Freund und engsten Mitarbeiter Rolf Adlers, dem das Buch gewidmet ist). Und er steht symbolhaft für das ganze Buch: in konzentrierter, genau gesetzter Sprache die Kernthemen der biopsychosozialen Medizin fassbar zu machen.

Peter Weibel, Bern
Psychodynamische Kurzund Fokaltherapie

\section{Theorie und Praxis}

Joachim Küchenhoff, unter Mitarbeit von Cordula Olshausen Küchenhoff Stuttgart: Schattauer; 2005.

Das Buch vermittelt einen Überblick über die meisten Konzepte der psychoanalytischen Kurztherapie. Aus diesen werden Grundprinzipien für die Ausgestaltung der psychoanalytischen Kurztherapie (PAKT) entwickelt, wobei diese Prinzipien immer wieder in Relation zur Psychoanalyse und zu klinischen Anforderungen diskutiert werden. Interessant sind dabei etwa die Reflexionen über die Bedeutung der (kurzen) Zeit. Im Teil über die PAKT werden verschiedene Anwendungsformen dargestellt. Abschliessend werden ausführliche Fallvorstellungen in unterschiedlichen Settings referiert.

Ein besonderer Stellenwert wird dem Thema Therapiefokus eingeräumt, der als eine Art Leuchtspur und Organisationsprinzip in der Gestaltung der Therapie verwendet wird. Er wird aus verschiedenen Elementen zusammengesetzt (z.B. aktuelle Klinik, Struktur, Dynamik, Übertragung, Biographie). Als Referenzsystem wird die OPD (operationalisierte psychodynamische Diagnostik) verwendet. Dies ermögliche eine vereinfachende und differenzierte Diagnostik und erlaube, insbesondere bei strukturellen Störungen, die Ziele genau anzugeben. Zudem erleichtere die OPD auch die vergleichende Forschung.

Interessant ist, dass der Autor die PAKT auch auf strukturelle Störungen anwendet für $\mathrm{Pa}$ tienten mit Persönlichkeitsstörungen und geringer Integration ihrer psychischen Struktur. Dabei geht es ihm immer auch um eine Verbindung mit dynamischen Gesichtspunkten: «Selbst wenn Strukturmerkmale per se als überdauernd bezeichnet beziehungsweise sich träge verändernd angenommen werden, unterliegen sie dennoch einer Dynamik.» Die Verbindung von Struktur und Dynamik erlaubt es, verschiedene Anwendungen $\mathrm{zu}$ unterscheiden, welche insbesondere für stationäre und teilstationäre Bedingungen sinnvoll sind.

Hervorzuheben sind auch die Überlegungen zur Indikation. Es geht hier um komplexe Fragen: Aus psychoanalytischem Blickwinkel sollten zwar Konfliktlösungen und Strukturveränderungen erreicht werden, aus äusseren praktischen Gründen steht aber nicht immer die entsprechende Zeit zur Verfügung. Zudem können aus Gründen der Störungen sowie der therapeutischen Prozesse fraktionierte kurztherapeutische Angebote besonders adäquat sein. Der Autor verwendet hierzu den Begriff der «adaptativen Indikationsstellung». Diese orientiert sich nicht allein an der Störung, sondern berücksichtigt Faktoren des Therapeuten, des therapeutischen Umfeldes, der Ressourcen und Zielsetzungen des Patienten. «Die Auswahlkriterien richten sich nach dem Therapieanspruch in einer bestimmten Situation, nicht am Anspruch, eine Störung zu heilen.»

Das Buch ist übersichtlich gegliedert und arbeitet mit prägnanten Zusammenfassungen. Es gleicht einem Reiseführer, der eine Gegend von verschiedensten Seiten her beleuchtet, ohne aber eine ganz bestimmte Reiseroute vorzuschreiben. Obwohl in kurzer Form und wie gut verständlich die wesentlichen Begriffe der psychoanalytischen Therapeutik auch abgehandelt werden, ist die Handhabung der PAKT - insbesondere die Formulierung eines Fokus - eher eine Methode für erfahrene Therapeuten. Auf wenigen Seiten werden am Schluss rund 50 Therapieregeln dargestellt. Diese ergeben eine wertvolle Orientierung, verlangen für ihre Aneignung aber eine vertiefende Auseinandersetzung.

Rudolf Balmer, Basel 